\title{
Synthesis of Phase-locked Loop System Structure with Adaptation Based on Combined-maximum Principle
}

\author{
Andrey Aleksandrovich Kostoglotov ${ }^{1}$, Sergey Valerievich Lazarenko ${ }^{2}$, Dmitriy Sergeevich Andrashitov ${ }^{3}$ and Igor \\ Vladimirovich Deryabkin ${ }^{1}$ \\ ${ }^{1}$ Rostov State Transport University, Department of Informatics, Rostov-on-Don, Russian Federation \\ ${ }^{2}$ Don State Technical University, Department of Radio Engineering and Electronics, Rostov-on-Don, Russian Federation \\ ${ }^{3}$ Moscow Vitte University, Department of Mathematics and Informatics, Moscow, Russian Federation
}

\begin{abstract}
The synthesis of the structure of the phase-locked loop system is performed based on the generalized power function maximum condition. The system effectiveness in the lock-on mode is studied via numerical simulation. Compared to the classical one phase-locked loop system with RC-filter this solution is characterized by the higher stability with respect to external influences and by reduced phase error in the steady-state.
\end{abstract}

\section{Introduction}

The phase-locked loop (PLL) systems have a wide range of applications in communications technology. The design of PLL is often brought to choosing a structure and parameters of the system at a given initial data and conditions of usage so it would satisfy the required criteria in the best way. PLL is now widely used in the systems described by the nonlinear differential equations of second and higher orders. The design of such PLL needs to determine the conditions for stability of the required mode and the qualitative characteristics of the stabilization process. Many methods providing the synthesis problem solution are known. But they implement a rational but not optimal solutions. Therefore, building an optimal compensating circuit for the phaselocked loop system is actual problem.

In this paper the problem of synthesis of a compensating circuit is formulated as an optimal control problem [1]. As initial system we consider PLL based on single-section RC-filter [2].

The aim of this paper is synthesis and study of the phase-locked loop system with adaptation to external influences on the basis of the combined-maximum principle.

\section{Problem formulation}

The basic assumptions of dynamics, the Newton's axioms and d'Alembert's principle, allow to formulate the laws of motion in the form of differential equations, which are an integral part of the formulation of optimization problems. But the equal possibilities are provided by the variational principles which determine the stationary properties of some quantities depending on the system parameters.

Let us consider the holonomic controlled dynamical system. Its action integral has the form [3]

$$
R=\int_{0}^{t_{1}}(T+A) d t
$$

where $T=\frac{1}{2} \sum_{s, k=1}^{n} a_{s k} \dot{q}_{s} \dot{q}_{k} \quad$ is the kinetic energy; $\mathbf{q}=\left[q_{1}, \ldots, q_{n}\right]^{\mathrm{T}}$ is the vector of generalized coordinates; $a_{s k}$ is the inertia factors; $A=\int_{\mathbf{q}(0)}^{\mathbf{q}\left(t_{1}\right)} \sum_{s=1}^{n} Q_{S} d q_{S}$ is work of the generalized external forces; $\mathbf{Q}=\left[Q_{1}(\mathbf{q}, \dot{\mathbf{q}}, \mathbf{u}), \ldots, Q_{1}(\mathbf{q}, \dot{\mathbf{q}}, \mathbf{u})\right]^{\mathrm{T}}$ is the vector of generalized forces which is continuous with respect to all its variables; $\mathbf{u}=\left[u_{1}, \ldots \mu_{m}\right]^{\mathrm{T}}$ is the control vector; $\mathbf{q}(0)$, $\mathbf{q}(t)$ are the initial and current values of the generalized coordinates vector; $\mathrm{n}=\operatorname{dim} \mathbf{q} \geq \mathrm{m}=\operatorname{dim} \mathbf{u}$; ${ }^{\mathrm{T}}$ is the symbol of matrix transposition, and the dot denotes time derivative.

Now we require that when the system moves from initial state

$$
\begin{aligned}
& t=0, \\
& \mathbf{q}(0)=\left[q_{10}, \ldots, q_{n 0}\right]^{\mathrm{T}}, \dot{\mathbf{q}}(0)=\left[\dot{q}_{10}, \ldots, \dot{q}_{n 0}\right]^{\mathrm{T}}
\end{aligned}
$$

to the final state 


$$
\begin{aligned}
& t=t_{1}, \\
& \mathbf{q}\left(t_{1}\right)=\left[q_{11}, \ldots, q_{n 1}\right]^{\mathrm{T}}, \dot{\mathbf{q}}(0)=\left[\dot{q}_{11}, \ldots, \dot{q}_{n 1}\right]^{\mathrm{T}}
\end{aligned}
$$

under control $\boldsymbol{u}$ the relation similar to the HamiltonOstrogradski principle for action integral (1) is true:

$$
\delta R=\int_{0}^{t_{1}}\left(\delta T+\delta^{\prime} A\right) d t=0
$$

where $\delta T$ is variation of $T, \delta^{\prime}$ is elementary increment [4], and

$$
\delta^{\prime} A=\sum_{S=1}^{n} Q_{S} \delta q_{s}
$$

Note that this requirement lets us state that the Lagrange equations of the second kind are true. For a holonomic system they can be written in the form [3]:

$$
\frac{d}{d t}\left(\frac{\partial T}{\partial \dot{q}_{S}}\right)-\frac{\partial T}{\partial q_{S}}=Q_{S}, s=\overline{1, n} .
$$

Let a constant sign, scalar, continuous with its partial derivatives on the the entire domain function $F(\mathbf{q})$ is given.

Formulation of the optimal control problem is as follows [5]: we need to find the vector-functions $\mathbf{Q}(\mathbf{q}, \dot{\mathbf{q}})$ and $\mathbf{q}$ which minimize the functional

$$
J_{1}=\int_{0}^{t_{1}} F(\mathbf{q}) d t \rightarrow \min
$$

with condition (4) and the restriction

$$
\mathbf{Q} \in \overline{\mathrm{G}}_{Q}
$$

where $\overline{\mathrm{G}}_{Q}$ is closed set of valid controls in the space of functions which are sectionally continuous in the finite time interval $\left[0, t_{1}\right]$.

Let us consider the extended functional of the following form:

$$
J=\int_{0}^{t_{1}}(F(\mathbf{q})+\lambda(T+A)) d t,
$$

where $\lambda$ is the Lagrange factor.

Let $\hat{\mathbf{Q}} \in \overline{\mathrm{G}}_{Q}$ be an arbitrary possible generalized force. Then if $\widetilde{\mathbf{Q}}$ minimizes functional (9) it is necessary that its variation is nonnegative $\delta J \geq 0$ for any possible variations $\delta \mathbf{Q}$.

Let us take into consideration the function $\Phi(\mathbf{q}, \dot{\mathbf{q}}, \mathbf{Q}, \lambda)$

$$
\Phi=\sum_{s=1}^{n}\left[\lambda Q_{s}(\mathbf{q}, \dot{\mathbf{q}}, \mathbf{u})+V_{s}(\mathbf{q})\right] \dot{q}_{s},
$$

where $V_{S}=\delta^{\prime} F / \delta q_{S}$. The physical meaning of $\Phi$ is a power of actual generalized forces $Q_{S}=Q_{S}(\mathbf{q}, \dot{\mathbf{q}}, \mathbf{u})$ and fictitious generalized forces $V_{S}(\mathbf{q}, \dot{\mathbf{q}})=\delta^{\prime} F / \delta q_{S}$ which are defined by the form of the given functional. The function $\Phi(\mathbf{q}, \dot{\mathbf{q}}, \mathbf{Q}, \lambda), \lambda \in \mathrm{R}$, is continuous with respect to the set of variables and is defined in the interval

$$
(\mathbf{q}, \dot{\mathbf{q}}, \mathbf{Q}, \lambda) \in \Omega \times \overline{\mathrm{G}}_{Q} \times \mathrm{R},
$$

where $\Omega$ is some area which is bounded in the space of functions and continuous within the time interval $\left[0, t_{1}\right]$. The area represents the set of solutions to equation (5). Boundedness of the area $\Omega$ comes from a continuous dependence of solutions to equation (5) with respect to the generalized force.

Theorem [1, 4]. If the generalized force $\tilde{\mathbf{Q}}(t)$ minimizes functional (7) under conditions (2), (3), (4), (6), then there exists such constant Lagrange factor $\lambda$, that for any $t \in\left[0, t_{1}\right]$ function $\Phi$ of the variables $(\mathbf{q}, \dot{\mathbf{q}}, \mathbf{Q}) \in \Omega \times \overline{\mathrm{G}}_{Q}$ reaches at the point $(\widetilde{\mathbf{Q}}(t), \widetilde{\mathbf{q}}(t), \dot{\widetilde{\mathbf{q}}}(t))$ its maximum

$$
\Phi(\widetilde{\mathbf{Q}}, \widetilde{\mathbf{q}}, \dot{\widetilde{\mathbf{q}}})=\max _{\mathbf{Q}(\mathbf{q}, \dot{\mathbf{q}}) \in \overline{\mathrm{G}}_{Q}} \Phi(\mathbf{q}, \dot{\mathbf{q}}, \mathbf{Q}, \lambda)
$$

\section{The synthesis of the phase-locked loop structure}

Choosing the single-section RC-filter as a loop filter we get the nonlinear differential equation describing the phase-locked loop (PLL) system [3]:

$$
T \frac{d^{2} \varphi}{d t^{2}}+\frac{d \varphi}{d t}+\Omega_{\mathrm{y}} F(\varphi)=\omega_{\mathrm{H}} .
$$

where $\varphi(t)=\varphi_{\mathrm{c}}(t)-\varphi_{0}(t)$ is difference between phases of signal and adjustable oscillator; $F(\varphi)$ is output of the phase discriminator, $\omega_{\mathrm{H}}=\dot{\varphi}_{\mathrm{c}}\left(t_{0}\right)-\dot{\varphi}_{0}\left(t_{0}\right)$ is difference between cyclic frequency of signal and adjustable oscillator at the initial time $t_{0},\left(\varphi\left(t_{0}\right), \dot{\varphi}\left(t_{0}\right)\right)$ are initial conditions. This equation can be represented in the form of the Lagrange equation of the second kind.

Therefore, we can formulate the problem to find the adaptive control for PLL system under conditions of external influences based on the combined-maximum principle method.

We now consider the problem to find $U(\varphi, \dot{\varphi})<|U|$ providing the transition of the dynamic system

$$
T \frac{d^{2} \varphi}{d t^{2}}+\frac{d \varphi}{d t}+\Omega_{\mathrm{y}} F(\varphi)=\omega_{\mathrm{H}}+U
$$

from any initial arbitrary point of the phase space $\left(\varphi\left(t_{0}\right), \dot{\varphi}\left(t_{0}\right)\right)$ to the final point $(0,0)$, so that 


$$
J=\frac{1}{2} \int_{t_{0}}^{t_{1}} \varphi^{2} d t \rightarrow \min .
$$

In accordance with eq. (12) from the condition of maximum of the function $\Phi=[\lambda U(\varphi, \dot{\varphi})+\varphi] \dot{\varphi}$ with restrictions $U(\varphi, \dot{\varphi})<|U|$, we get the following PLL [5]:

$$
U=-\Omega_{\mathrm{y}} \lambda^{-1}|U| \operatorname{sign}\left(\frac{D|\dot{\varphi}| \dot{\varphi}}{L|\varphi|+\varepsilon} \frac{T}{\Omega_{\mathrm{y}}}+\varphi\right),
$$

where $\lambda^{-1}$ is undefined Lagrange factor, $L$ is a constant depending on the form of the switching line, $\varepsilon, D$ are constants which define correspondingly the shift of switching lines and diameter of the ellipse in the phase space of states of the PPL system.

The PLL structure is defined by the following equation:

$$
\begin{aligned}
& T \frac{d^{2} \varphi}{d t^{2}}+\frac{d \varphi}{d t}+\Omega_{\mathrm{y}} F(\varphi)=\omega_{\mathrm{н}}- \\
& -\Omega_{\mathrm{y}} \lambda^{-1}|U| \operatorname{sign}\left(\frac{D|\dot{\varphi}| \dot{\varphi}}{L|\varphi|+\varepsilon} \frac{T}{\Omega_{\mathrm{y}}}+\varphi\right) .
\end{aligned}
$$

During numerical experiment we have obtained the solutions to the differential equations describing the PLL system with the RC-filter (13) and the PLL system with the autoregulation loop (16) on the basis of the combined-maximum principle. The phase discriminator is:

$$
F(\varphi)=\sin (\varphi)
$$

The simulation has been performed for the following values: $\mathrm{T}=0,014 \mathrm{c} ; \Omega \mathrm{y}=21 \mathrm{paд} / \mathrm{c} ;|\mathrm{U}|=5 ; \lambda-1=1$; $\varepsilon=0,001 ; \mathrm{D}=1 ; \mathrm{L}=4$.

The Fig. 1 shows the lock-on process on the phase plane.

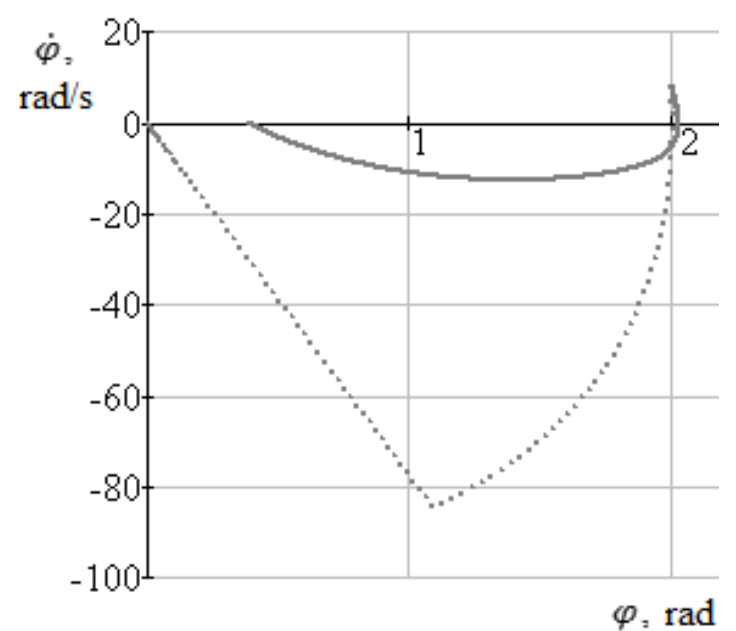

Figure 1. The phase plane of the PLL under consideration.

The Fig. 2 shows the dependence of the lock-on time $t_{3}$ for the considered PLL systems on the initial frequency mismatch $\dot{\varphi}\left(t_{0}\right)$ for three initial phases mismatches $\varphi\left(t_{0}\right)=0$ radian, $\varphi\left(t_{0}\right)=1$ radian, and $\varphi\left(t_{0}\right)=2$ radian.
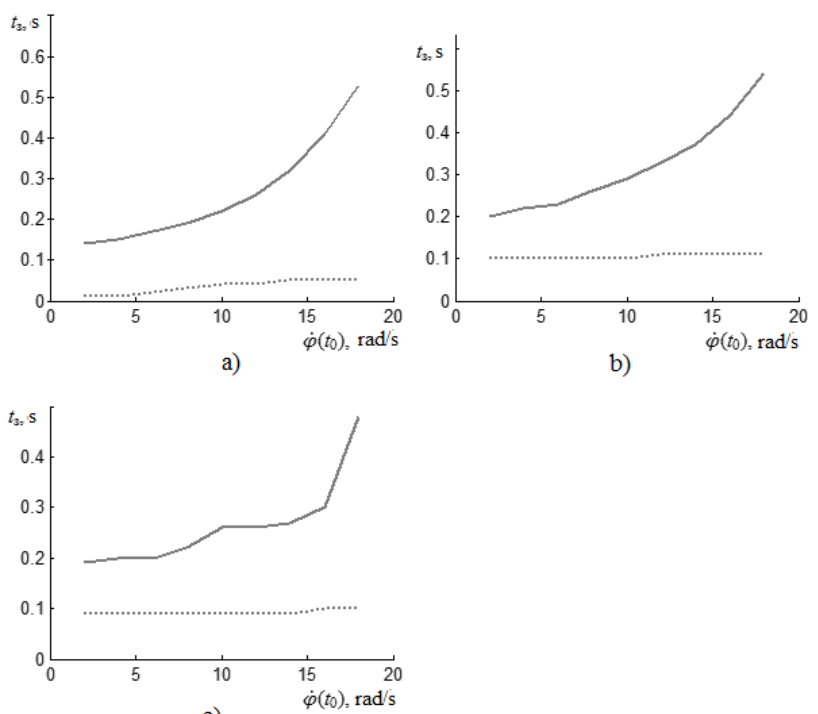

c)

Figure 2. The dependence of the lock-on time on the the initial frequency mismatch: a) at $\varphi\left(\mathrm{t}_{0}\right)=0$ radian; b) at $\varphi\left(\mathrm{t}_{0}\right)=1$ radian; c) при $\varphi\left(\mathrm{t}_{0}\right)=2$ radian.

These results shows that the lock-on time of the PLLcontrolled system using the combined-maximum principle method is approximately 2-6 times less and is almost independent on the initial conditions. For this PLL in the holding mode the steady phase difference $\varphi_{\mathrm{y}}=0$, unlike PLL with RC-filter for which $\varphi_{\mathrm{y}}=\arcsin (\omega \mathrm{H} / \Omega \mathrm{y})+2 \pi \mathrm{k}$. In addition for the PLL using the combined-maximum principle we see the lock-on possibility for the initial phases mismatches greater than for PLL with RC-filter (which demonstrates beats, see Fig. 3).

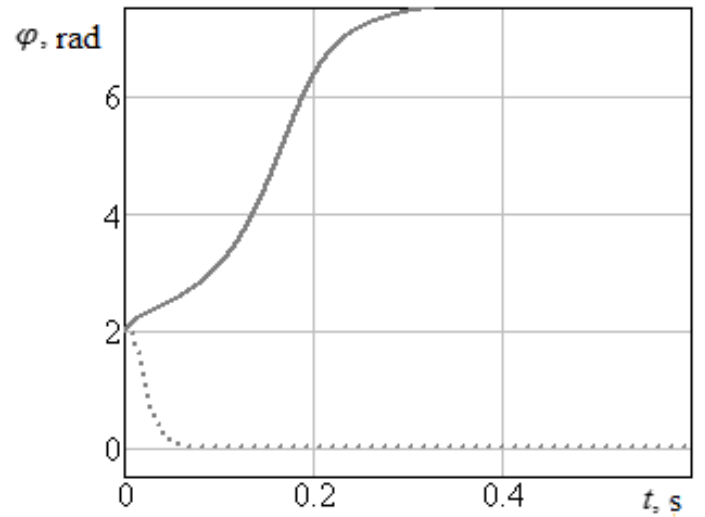

Figure 3. The dependence $\varphi(t)$ when the initial frequency mismatch with respect to the lock-on frequency band of the PLL system with RC-filter $\left(\varphi\left(t_{0}\right)=2 \operatorname{radian}, \dot{\varphi}\left(t_{0}\right)=\right.$ $22 \mathrm{radian} / \mathrm{sec}$ )

The simulation has been also performed with additional model external random interference with $D_{\varphi}=1 \cdot 10^{-4}$ radian $^{2}$. The results of the simulation are shown on Fig. 4. The numbers mean: 1 - the phase trajectory (16), 2 - the phase trajectory (12). The analysis 
of the phase portraits lets us declare the higher degree of resistance of the system (16) to external influences compared to (12) and the decrease of the phase errors in steady mode.

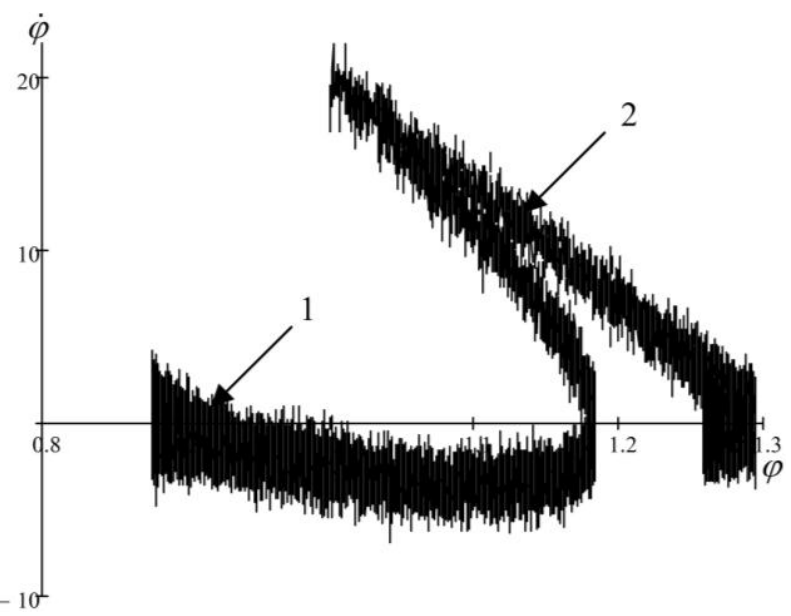

Figure 4. The phase portrait

We can getting alternative equation of PLL based on combined maximum principle

$$
\begin{aligned}
& T \frac{d^{2} \varphi}{d t^{2}}+\frac{d \varphi}{d t}+\Omega_{\mathrm{y}} F(\varphi)=\omega_{\mathrm{H}}- \\
& -\lambda^{-1}\left(\frac{T D|\dot{\varphi}| \dot{\varphi}}{L|\varphi|+\varepsilon}+\Omega_{\mathrm{y}} \varphi\right) .
\end{aligned}
$$

The results of modeling are illustrated on Fig. 5. Its demonstrate are capture process on a phase plane. There are the dotted line shows the solution corresponding to (13), and the solid (19). In this case, the system phaselocked loop with loop autoregulatory advantageously has a high resistance to accidental external interference in comparison with the system phase-locked loop with a simple RC-filter.

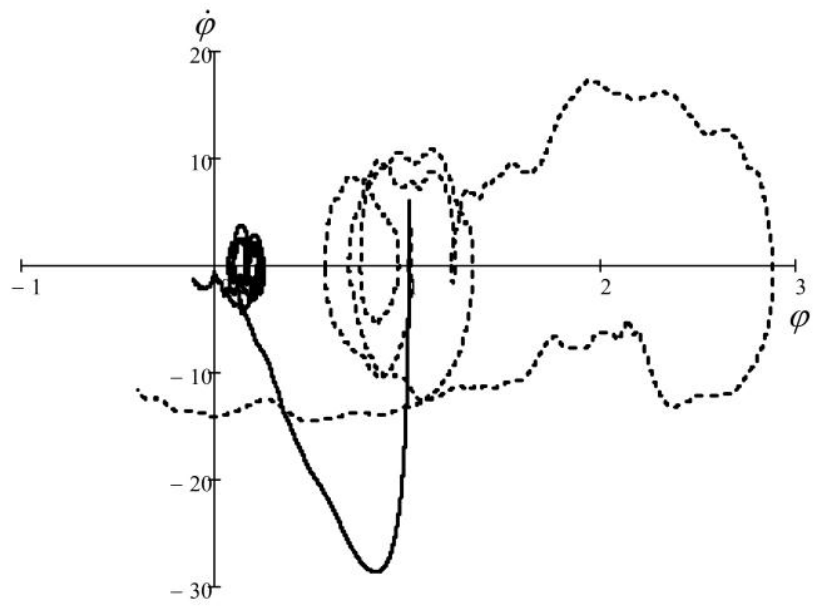

Figure 5. The phase portrait
The analysis of the functioning of the phase-locked loop system with adaptive feedback based on the combined maximum principle shows that the obtained solution can improve the efficiency of the classical scheme with respect to the criterion of minimal lock-on time and minimal synchronization errors. It has found out that this system allows to decrease the lock-on time 2-6 times approximately and to increase the PLL holding range 1.5 times compared to the single-section $\mathrm{RC}$-filter case. In the absence of observation noise the zero phase error is provided in the synchronization mode. This allows to use the obtained solution for constructing precision instruments to measure range and velocity.

\section{Conclusion}

Analysis of the results of mathematical simulation leads to the following conclusions:

1. The system reduces the lock-on time approximately 2-6 times compared to the PLL with the single-section RC-filter.

2. The system increases about 1.5 times the holding range compared to the PLL with the single-section RCfilter.

In addition, the study of operation of the synthesized phase-locked loop system under external influences has been carried out. The use of the system provides a higher stability when solving the phase lock-on problem and reduce the phase error in the steady mode compared to the classical phase-locked system.

\section{Acknowledgment}

The paper has been accomplished with the support of Russian Federal Property Fund grants No. 15-08-03798, 15-38-20835, 16-37-60034, 16-38-00665.

\section{References}

1. A.A. Kostoglotov, "Solution of Fuller's problem on the basis of the joint Pontryagin-HamiltonOstrogradskii principle," Automatic Control and Computer Sciences, 41, pp. 179 - 187 (2007).

2. V.V. Shahgil'djan, and A.A. Ljahovskij, Frequency phase-locked systems. Moscow: Svjaz' (1972).

3. A.I. Lur'e, Analytical mechanics. Moscow: Gos. Izd. Fiz.-Matem. Liter. (1961).

4. A.A. Kostoglotov, A.I. Kostoglotov, and S.V. Lazarenko, "Joint maximum principle in the problem of synthesizing an optimal control of nonlinear systems," Automatic Control and Computer Sciences, 41, pp. $274-281$ (2007).

5. A.A. Kostoglotov, A.I. Kostoglotov, S.V. Lazarenko, and B.M. Tsennykh, "Method of StructuralParametric Identification of Lagrangian Dynamic Systems in the Processing of Measurement Information," Measurement Techniques, 57, pp. 153 - 159 (2014) 